\title{
Motion of a Vector Particle in a Curved Space-time. IV. Asymptotical shape of caustic
}

\author{
A.T. Muminov \\ Ulugh Beg Astronomical Institute, Astronomicheskaya 33, Tashkent 100052 Uzbekistan \\ amuminov2002@yahoo.com \\ Z.Ya. Turakulov \\ Ulugh Beg Astronomical Institute, \\ Institute of Nuclear Physics, Ulughbek, Tashkent 102132 Uzbekistan
}

January 14, 2019

\begin{abstract}
The studies of influence of spin on a photon motion in a Schwartzschild spacetime is continued. In the previous paper [2] the first order correction to the geodesic motion is reduced to a non-uniform linear ordinary differential equation and the equation obtained has been solved by the standard method of integration of the Green function. If each photon draws a world line specified by this solution then light rays from infinitely distant source form a caustic which does not appear without the spin-gravity interaction. The goal of the present work is to obtain explicit form of caustic.

Keywords: Worldline of photon; Spin-gravitational interaction; Schwarzschild spacetime.
\end{abstract}

\section{Introduction}

In our recent works $[1,2]$ deviation of photon world line from a null geodesic was obtained in the first order approximation on spin-gravity interaction in Schwarzschild space-time. If all world lines form a parallel beam from an infinitely distant source, spin-gravitational interaction of photon should give the following effect. Since the world lines obtained do not lie wholly on one semi-plane of constant azimuthal angle $\varphi$ they do not cross the axis of symmetry on which the source is, but pass at some non-zero distance from it. Then some neighbohood of the back semi-axis is shadowed by the deviation. The shadow has certain boundary formed by the envelope of the rays, hence, this surface appears as caustic of this family of curves. The goal of the present work is to find out the shape of the surface.

To obtain the shape of the surface we need to consider some details of calculations of the reference geodesic and our first-order approximation. Therefore it is useful to recall the techniques and the denotions used. We use the standard denotions: 
coordinates for the Schwartzschild space time are $\{t, r, \theta, \varphi\}$ and null geodesics lying wholly on the $\theta=p i / 2$ equatorial "plane" are presented in the parametric form

$$
t=-\frac{\partial R}{\partial E}, \quad \varphi=\varphi_{0}+\frac{\partial R}{\partial L}, \quad \theta \equiv \pi / 2
$$

where

$$
R(r)=-\varepsilon(t) \int_{r_{0}}^{r} \frac{\sqrt{E^{2} r^{2}-L^{2}\left(1-r_{g} / r\right)}}{r\left(1-r_{g} / r\right)} d r,
$$

$\varepsilon(t)=\operatorname{signum} t$ which follows from the corresponding solution $\Psi=E t-L \varphi+R(r)$ of the Hamilton-Jacobi equation $\langle d \Psi, d \Psi\rangle=0$. The constants are chosen such that under $t=0$ each geodesic reaches the minimal value of $r, r=r_{0}$. Then under $t \rightarrow-\infty$ the geodesic approaches the starting point at the source and under $t \rightarrow+\infty$ it runs away to the flat asymptotics. We put $\left.\varphi\right|_{t \rightarrow-\infty}=0$, so that $\varphi_{0}=\left|\frac{\partial R}{\partial L}\right|_{r \rightarrow \infty}$. It must be noted that unlike action of a massive particle which can be used as a parameter on the geodesic, that of massless one $\Psi$ takes constant values on null geodesics in question. Therefore, $\Psi$ cannot be used as a parameter on the geodesics.

The form of 0 -geodesics determined by squaring (1) is given in terms of hyperelliptic functions. In fact, we do not need the exact expressions because in the commonplace approximation we employ: $r_{g} \ll D$ where $D=L / E$ stands for the impact parameter, all expressions simplfy (see, for example, Ref[3]). The radial part (2) of the action function $\Psi$ can be represented as

$$
R(s)=-\varepsilon(s) \int_{r_{0}}^{r} \sqrt{\frac{1}{\left(1-r_{g} / r\right)^{2}}-\frac{D^{2}}{r^{2}\left(1-r_{g} / r\right)}} d r .
$$

Following [3] we substitute

$$
r\left(r-r_{g}\right)={r^{\prime}}^{2}, \quad \text { so that } \quad r^{\prime} \approx r-r_{g} / 2 .
$$

Then the function $R(s)$ becomes

$$
R(s) \approx-\varepsilon(s) \int_{r_{0}-r_{g} / 2}^{r-r_{g} / 2} \sqrt{1+\frac{2 r_{g}}{r^{\prime}}-\frac{D^{2}}{r^{\prime 2}}} d r^{\prime} .
$$

Moreover, we have

$$
r^{\prime 2}+2 r_{g} r^{\prime}-D^{2}=x^{2}-B^{2}, \quad B^{2}=D^{2}+r_{g}^{2}, \quad x=r^{\prime}+r_{g} \approx r+r_{g} / 2 .
$$

Another substitution in the integrand yields:

$$
R(s) \approx-\varepsilon(s) E \int_{D}^{r+r_{g}} \frac{\left(x^{2}-D^{2}\right)^{1 / 2}}{x-r_{g}} d x .
$$

This result allows to represent the shape of light ray approximately in analytical form considered below 


\section{Analytical representation of the shape of the ray}

We see that the factor annullating the integrand at the lower limit is separated and now we can safely expand another factor putting $r_{g} / x \leq r_{g} / D \ll 1$ that gives:

$$
R(s) \approx-\varepsilon(s) E \int_{D}^{r+r_{g}} \frac{\left(x^{2}-D^{2}\right)^{1 / 2}}{x}\left(1+r_{g} / x\right) d x .
$$

It is seen that the integral admits analytical representation.

The shape of the null geodesic is determined by dependence of angle $\varphi$ on $s$ given by eq. (1) due to Hamilton-Jacobi theorem. Substituting eq. (3) into the eq. for $\varphi$ we obtain:

$$
\varphi \approx \varphi_{0}+\varepsilon \int_{D}^{r+r_{g} / 2}\left\{\frac{D}{x \sqrt{x^{2}-D^{2}}}+\frac{r_{g} D}{x^{2} \sqrt{x^{2}-D^{2}}}\right\} d x
$$

The constant parameter $\varphi_{0}$ can be eliminated by corresponding choice of initial value of this coordinate: $\varphi(-\infty)=0$ accepted in our previous work [2]. This choice yields the following explicit form of $\varphi$ as function of the variable $s$ :

$$
\varphi(s) \approx \pi / 2+r_{g} / D+\varepsilon(s)\left\{\arccos \frac{D}{x}+\frac{r_{g} \sqrt{x^{2}-D^{2}}}{D x}\right\}_{x=r+r_{g} / 2} .
$$

Asymptotical shape of the caustic to be found is formed by $s>0$ parts of the geodesics:

$$
\varphi(s>0) \approx \pi / 2+r_{g} / D+\arccos \frac{D\left(r-r_{g} / 2\right)}{r^{2}}+\frac{r_{g} \sqrt{r^{2}-D^{2}}}{D r} .
$$

It was shown in our previous work [2] that in the first order approximation the geodesics deviate only in $\partial_{\theta}$ direction. The deviation is specified by the vector of the deviation $\delta \vec{x}=\delta x^{2} r^{-1} \partial_{\theta}$. Its only non-zero component for the outgoing branch of the trajectory $(s>0)$ has the following asymptotical behavior under $r \rightarrow \infty$ :

$$
\delta x^{2} \approx \frac{r_{g} r}{E D^{2}}
$$

where we have introduced local Cartesian coordinate $x^{2}$ such that $d x^{2}=r d \theta$. Finally, approximate world line of photon is given by the reference geodesic and the small vector of deviation just provided.

\section{The shape of asymptotical part of the caus- tic}

Since the shape of caustic of stationary beam of light is stationary as well as the Schwarschild space-time itself, we do not need the time coordinate on the world line of given photon and can consider only the shape of separate light ray in the space endowed with standard spherical coordinates $\{r, \varphi, \theta\}$.

The envelope of a beam of such rays is just the caustic to be found. Besides, axial svmmetrv of the envelope allows to represent it by the shape of curve along which 
the surface crosses equatorial semi-plane $0 \leq \varphi \leq \pi / 2, \theta=\pi / 2$. In other words, to obtain the desired surface it suffices to find out the distance between envelope and the axis $\rho^{2}=r^{2} \sin ^{2}(\varphi)+\left(\delta x^{2}\right)^{2}$ as function of the coordinate $r$. This task reduces the problem of constructing the envelope of family of curves on the semi-plane

$$
\rho(r, D)=\sqrt{r^{2} \sin ^{2}(\varphi)+\left(\delta x^{2}\right)^{2}},
$$

where each curve is labeled with certain value of the impact parameter $D$. Value of $\varphi$ is given by (4). It is convenient to use the coordinates $\{r, \rho\}$ in the semi-plane instead of the standard ones $\{r, \varphi\}$.

Appearance of caustic can be explained geometrically as follows. Due to the spin-gravitational interaction the rays instead of crossing the axis pass at distance $\rho$ from it. The rays which are asymptotically straight lines, under given value of the impact parameter $D$ lie on one-sheet hyperboloids whereas the geodesics do on cones with same axis. Unlike the cones, the hyperboloids of distinct $D$ constitute a family which has an envelope. In two-dimensional picture they are hyperbolas whereas the geodesics are straight lines incident to the axis. The hyperbolas have an envelope we are constructing. In this section we simplify the task assuming that the envelope of hyperbolas almost coincides with the curve on which their points closest to the axis lie. We call this curve "simplified" caustic and first, we explore it. Afterwards we show that in our approximation this curve coincides with the genuine caustic constructed in classical approach.

Since all the results are obtained under assumption that the value $r_{g} / D$ is small, the impact parameter is to be taken sufficiently big $D \gg r_{g}$ that allows to obtain only asymptotical behavior of the caustic. To do it we need explicit form of the expression for the function $\sin \varphi(r)$ in our approximation. Straightforward substitution of the equation (4) yilds:

$$
\sin (\varphi) \approx D\left(1 / r+r_{g} / r^{2}\right)-2 r_{g} / D \approx \frac{D}{r}-\frac{2 r_{g}}{D}
$$

It is convenient to represent the envelope by the curve $r=r_{x}(D), \rho=\rho\left(r_{x}(D), D\right)$, where $r_{x}(D)$ is the value of the coordinate $r$ under which the ray with impact parameter $D$ crosses the axis. To find it we solve the equation

$$
\sin \varphi(r, D)=0
$$

which due to equation (7) reduces to

$$
r_{x}=\frac{D^{2}}{2 r_{g}}
$$

This allows to express the variable $\rho$ in the neighborhood of the crossing point $r=r_{x}$ :

$$
\rho=\delta x^{2}\left(r_{x}\right)=\frac{r_{g}}{E D^{2}} \cdot \frac{D^{2}}{2 r_{g}}=\frac{1}{2 E}=\frac{\lambda}{4 \pi},
$$

where $\lambda$ is wavelength. In other words, asymptotically the envelope becomes a cylinder of radius which disappears in astrophysical scales. 


\section{Classical approach}

Since simplified caustic is too narrow to be observed it is necessary to obtain the genuine caustic. The shape of the genuine caustic can be extracted from shape of envelope of curves (6) on which the following determinant vanishes:

$$
0=\left|\begin{array}{ll}
\frac{\partial \rho}{\partial r} & \frac{\partial \rho}{\partial D} \\
\frac{\partial r}{\partial r} & \frac{\partial r}{\partial D}
\end{array}\right|=\left|\begin{array}{cc}
\frac{\partial \rho}{\partial r} & \frac{\partial \rho}{\partial D} \\
1 & 0
\end{array}\right|=-\frac{\partial \rho}{\partial D} .
$$

Indeed, if we use the parameter $D$ as a coordinate instead of $\rho$, each curve is given by $D=$ const. Since the curves have an envelope the Jacobian of the transformation vanishes on it, consequently, the envelope to be found is zero of the determinant. Thus, the equation of the envelope can be written as follows:

$$
\frac{\partial \rho^{2}}{\partial D}=0
$$

It is seen that under $\delta x^{2}=0$ no envelope appears and since $\delta x^{2} \approx r_{g} r / E D^{2}$ the envelope can appear only at distances of order (8). Referring to the equations $(5,6,7)$ we have:

$$
\rho^{2} \approx \frac{r_{g}^{2} r^{2}}{E^{2} D^{4}}+D^{2}+\frac{4 r_{g}^{2} r^{2}}{D^{2}}-4 r_{g} r
$$

Now, substituting the expression into the equation (10) we obtain:

$$
0=\frac{\partial \rho}{\partial D} \approx-4 \frac{r_{g}^{2} r^{2}}{D^{3}}+2 D-8 \frac{r_{g}^{2} r^{2}}{D^{3}} .
$$

Taking account that $1 / E \sim \lambda \ll D$ we have finally the same result as in the equation (8):

$$
r^{2} \approx \frac{D^{4}}{4 r_{g}^{2}}
$$

for $r$ being the coordinate of point of intersection of the ray and the axis.

\section{$5 \quad$ Angular size of the caustic}

Usually, angular size of an object is specified by the angle under which the rays from it diverge on the axis. In this sense the caustic has zero angular size because its shape is cylindric. However, the rays are not parallel on it, consequently, some caustic must be seen. Below we calculate the angle under which they pass near the axis. Since the rays are straight near the crossing point the angle is $\delta \phi=\left.r \frac{\partial \varphi}{\partial r}\right|_{r=r_{x}}$. Substituting this into the equation (4) to the above expression gives:

$$
r \frac{\partial \varphi}{\partial r} \approx \frac{D}{\sqrt{r_{x}^{2}-D^{2}}} \approx \frac{D}{r_{x}}=\frac{2 r_{g}}{D}=\sqrt{\frac{2 r_{g}}{r}} .
$$

The angular size is doubled angle $\delta \varphi$, namely, $2 \sqrt{2 r_{g} / r}$ where $r$ is coordinate of observer. This result seems to be somewhat unexpected because angular size of caustic obtained this way does not depend on the wavelength whereas in the limit of zero wavelength the caustic does not appear at all. 
Since angular size of the caustic obtained this way is valid for all wavelengths it can be obtained also for geodesics which expose no spin gravitational interaction. The angle in question apparently if formed by geodesics focused onto the axis and must be observed because any telescope detects only direction from which radation comes. Naturally, radiation in this case comes from directions which consitute a cone which must be seen as a bright ring. However, this image has nothing to do with spin-gravitational effect and exposes only the fact of lensing. As for the spin-gravitational interaction, it does not produce any special observable effect but, probably, some diffusion of the bright ring with dependence of the diffusion on the wavelength.

\section{Conclusion}

This is the closing work of the series started with the article [4] in which Papapetrou equation was derived for a massless spining vector particle. In the work [1] derivation was slightly improved and an attempt was made to obtain an approximate solution of the equation. The final version of derivation of Papapetrou equation from Lagrangian of electromagnetic field is presented in our work [5]. In our work [2] it was shown that the method used in the work [1] is valid only on the first half of the photon world line and another method, valid on the whole world line is proposed. The new method allows one to obtain the whole world line as a small deviation from the reference geodesic.

All this work was completed with the purpose to explain why black holes appear in observations as bright rings. We hoped to prove that these rings are images of caustics formed by photons inclined from geodesics by the spin-gravitational interaction. This would be another test of general relativity. In fact, it is not so. As was shown above, caustics produced by this interaction are asymptotically too narrow and have actually zero angular size, therefore, is not observable. Instead, there exists ring-shaped image of another nature which has nothing to do with spin-gravitational interaction. Angular size of this image does not depend on the wavelength and exposes only focusing of geodesics. As for the spin-gravitational interaction, it can only give a subtle spectroscopic effect on the image.

\section{References}

[1] Turakulov Z Ya, Safonova M, Motion of a Vector Particle in a Curved SpaceTime. II. First-Order Correction to a Geodesic in a Schwarzschild background. Mod Phys Lett A 20 (2005) 2785

[2] Turakulov Z Ya, Muminov A T, Motion of a Vector Particle in a Curved SpaceTime. III. Development of Techniques of Calculations/Mod Phys Lett A21 n 26 (2006) 1981

[3] Landau, Lev D. and Lifshitz, Evgeny M. (1971). Classical Theory of Fields (3rd ed.). London: Pergamon.

[4] Turakulov Z Ya, Safonova M, Motion of a Vector Particle in a Curved SpaceTime. I. Lagrangian Approach/Mod Phys Lett A18 (2003) 579

[5] Turakulov Z. Ya, Muminov A. T, Electromagnetic field with constraints and Papapetrou equation. Zeitschrift fur Naturforschung 61a. 146 (2006) 